\title{
A Low-Cost Electrochemical Metal 3D Printer Based on a Microfluidic System for Printing Mesoscale Objects
}

\author{
Pengpeng Liu, Yawen Guo, Yihong Wu, Junyan Chen and Yabin Yang * \\ School of Materials Science and Engineering, Sun Yat-sen University, Guangzhou 510275, China; \\ liupp27@mail2.sysu.edu.cn (P.L.); guoyw26@mail2.sysu.edu.cn (Y.G.); wuyh55@mail2.sysu.edu.cn (Y.W.); \\ chenjy96@mail2.sysu.edu.cn (J.C.) \\ * Correspondence: yangyabin@mail.sysu.edu.cn
}

\begin{abstract}
For the additive manufacturing (AM) of metal objects, the powder-based fusion (PBF) method is routinely utilized to fabricate macroscale parts. On the other hand, electrochemical additive manufacturing (ECAM), in which metallic structures are deposited through the electrochemical reduction of metal ions, is a promising technique for producing micro- and nanoscale objects. However, a gap exists in terms of fabricating mesoscale objects within the current AM techniques. The PBF method is limited by fabrication precision due to pronounced residual stresses, and most current ECAM systems are difficult to scale up to print mesoscale objects. In the present paper, the novel design of a low-cost ECAM 3D printer based on a microfluidic system is proposed for fabricating mesoscale metal parts. The meniscus-guided electrodeposition approach is utilized, in which a meniscus is formed between the print head and substrate, and electrodeposition is confined within the meniscus. A 3D object is fabricated by the meniscus moving with the print head according to the programmed pattern and the material subsequently being deposited at the designated locations. The key to the proposed design is to maintain a mesoscale meniscus, which normally cannot be sustained by the electrolyte surface tension with a print nozzle having a mesoscale diameter. Therefore, a microfluidic system, called the fountain pen feed system, constituting a semi-open main channel and comb structure, was designed to maintain a mesoscale meniscus throughout the printing process. Two materials, copper and nickel, with various geometric shapes were attempted to print by the proposed ECAM system, and, during the printing process, both fluid leaking and meniscus breaking were completely prevented. Free standing tilted copper pillars with controlled angles were printed to show the ability of the proposed design in fabricating 3D structures. A copper circuit was also printed on a non-conductive substrate to demonstrate a possible application of the proposed ECAM system in the fabrication of functional electronics.
\end{abstract}

Keywords: electrochemical additive manufacturing; fountain pen feed system; metal 3D printer

\section{Introduction}

Additive manufacturing (AM) (3D printing), the method of creating geometrically complex 3D parts in a layer-by-layer manner, enables the fabrication of complex parts with high topological freedoms within a single manufacturing step. For metals, macroscale parts are usually fabricated using the powder-based fusion (PBF) method, which involves a high-energy external heat source such as a laser or electron beam to selectively melt metallic powders and fuse them together according to a computationally programmed pattern [1,2]. For microscale and nanoscale structures, electrochemical additive manufacturing (ECAM), in which metal ions are deposited as metal atoms at given locations 
by an electrochemical process, has been suggested as a promising approach to achieve excellent mechanical and electrical properties with extremely high resolution [3,4]. However, there is a gap in terms of cost-effective AM approaches to print mesoscale structures in the sub-millimeter range.

The resolution of the PBF method is limited by the powder diameter, which is in the range of 20-100 $\mu \mathrm{m}$ [5]. Additionally, high residual stress induced by thermal deformation [6,7] is pronounced in PBF, which considerably impairs the printing precision. For ECAM, two approaches are mainly utilized:

1. Localized electrodepotion (LED), for which a conductive substrate and an ultrasharp microelectrode are immersed into a bath of metal salt solution (electrolyte). Localized deposition of metals occurs through the electrochemical reduction when the microelectrode is very close to the substrate and an external electric field is applied between the microelectrode and substrate [8-10].

2. Meniscus-guided electrodeposition (MGED) [11], for which a micropipette that contains the metal salt solution is utilized. When the micropipette approaches the conductive substrate, a liquid meniscus is established. The electrodeposition is then confined to the small region of the meniscus when applying appropriate potential difference [12].

As in MGED, the printing process is operated in air, and the printed structures will not be contaminated or destroyed by submersion [11]. Seol et al. [4] demonstrated geometrically complex microarchitectures can be printed using the MGED approach by modulating the applied potential in two steps with different amplitudes and durations. By utilizing a pulsed potential, Behroozfar et al. [3] successfully printed nanotwinned-metals, which exhibit superior mechanical and electrical properties. Kim et al. $[13,14]$ developed an AFM-based nanofountain probe with the form of volcano tip, which is capable of delivering a meniscus at the sub-100 nm scale. This design is then successfully applied for multiple applications [15-18]. Besides, Falola et al. [19] introduced a wide range of elements that can be electrodeposited from aqueous electrolytes. Reiser et al. [20] printed bi-metal (Cu-Ag) pillars by controlling the electrohydrodynamic ejection of metal ions dissolved from the $\mathrm{Cu}$ or $\mathrm{Ag}$ anode. Chen et al. [21] developed a two-syringe system, one for copper and one for nickel, to fabricate thermally responsive $\mathrm{Cu}-\mathrm{Ni}$ strips. Ambrosi et al. [22] demonstrated how the materials (metal copper and polyaniline) to be deposited within a single syringe can be precisely controlled by tuning the applied potential.

Although these methods demonstrate they are valid alternatives to conventional ink-based or laser-based AM technologies, the products are mainly limited to micro- or nanoscale applications. Furthermore, expensive piezo-driven positioning stages are usually required to maintain a stable meniscus [22]. Therefore, a more economically efficient metal printing technology to fabricate large-scale products is highly needed. The key problem is how to maintain a stable mesoscale meniscus, which cannot be obtained merely by the surface tension of the liquid. Wang et al. [23] modified a syringe extruder to print $2 \mathrm{D}$ patterns of $\mathrm{Cu}_{2} \mathrm{O}$. A controlled volume of liquid electrolyte was ejected to form a meniscus between the print head and substrate. Kim et al. [24] introduced a suction nozzle at the print head to maintain a stable meniscus. Chen et al. [25] proposed an ECAM system based on low-cost stepper motor stages instead of expensive piezo-based stages. A nozzle with a diameter of $400 \mu \mathrm{m}$ was employed to increase the size of the meniscus considerably and thus improve the deposition rates. In order to maintain a stable meniscus with such a large diameter, they proposed inserting a porous sponge at the tip of the nozzle to adjust the electrolyte flow rate. They further improved their approach by using porous electrospun nanofiber mats at the tip instead of a sponge to print $\mathrm{Cu}-\mathrm{Ni}$ strips [21]. However, in their approach, the flow rate of the electrolyte solution was intrinsically controlled by the permeability of the porous material, which may vary during the printing process and hence cause certain difficulties in maintaining a stable meniscus. Moreover, precise control over insertion of the porous material at the desired nozzle position was also difficult. Nevertheless, they demonstrated that mesoscale meniscus-confined ECAM is possible. 
In the present paper, we propose a novel ECAM system design based on the MGED approach for printing mesoscale metal objects. A microfluidic system referred to as the fountain pen feed system was employed to maintain a stable mesoscale meniscus. With the proposed design, passive control of continuous electrolyte flow at the tip of the print head can be achieved without liquid leaking or breaking during the printing process, and thus the stability of the meniscus can be automatically adjusted by the fountain pen feed system. As there is no need for active control, the proposed fountain pen feed system is convenient to establish. Moreover, the proposed fountain pen feed system opens the door to controlling the profile of the meniscus with various designs of the microfluidic system. A fluid flow simulation performed on the commercial software Fluent was utilized to help to explain the mechanism of the fountain pen feed system. Two materials, copper and nickel, with various geometric shapes were attempted to print to demonstrate the feasibility of the proposed ECAM system. A printing process on a non-conductive substrate was also conducted to provide a possible application with the proposed design. The article concludes with a reiteration of the most salient points of the study.

\section{Design}

The proposed ECAM system is shown in Figure 1a. Movement of the print head is controlled by the $x-, y-$, and $z$ - stages. The design of the print head is schematically illustrated in Figure $1 \mathrm{~b}$. A microfluidic system, which is referred to as the fountain pen feed system, is proposed. This fountain pen feed system consists of the main channel part and the comb structure, which is further illustrated in details in Figure 2a. During the printing process, the electrolyte in the reservoir flows through the main channel to the tip by gravity and capillary action. At the same time, with the semi-open design of the main channel (as shown in the A-A cross-section of Figure 1b), air can also flow through the main channel into the reservoir, thereby providing a sufficient back pressure for continuous transport of electrolyte to the tip of the print head. When a positive potential is applied between the working electrode and substrate, the metal ions $M^{n+}$ within the meniscus are deposited on the substrate through the reduction of $M^{n+}+n e^{-}=M$. The meniscus moves with the print head according to a programmed path, and the materials are subsequently deposited at the designated locations to form the desired structure.

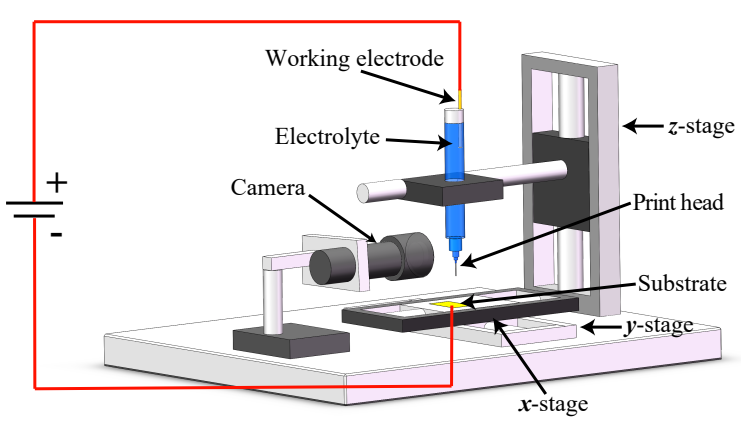

(a)

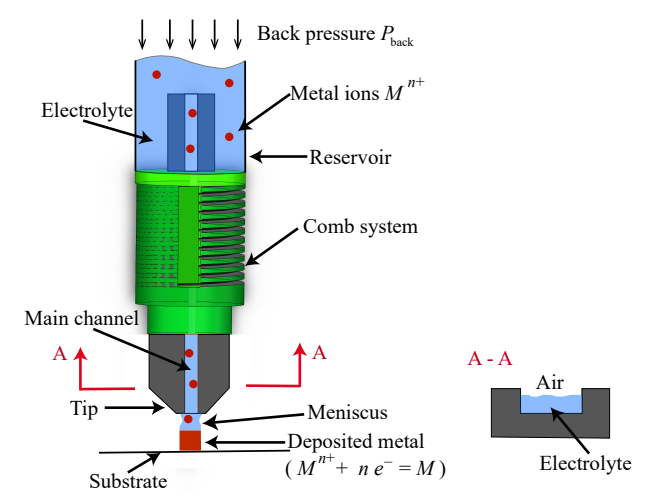

(b)

Figure 1. (a) Schematic illustration of the proposed ECAM system. (b) Detailed illustration of the print head, also referred to as the fountain pen feed system. The cross section A-A at the tip of the main channel is shown in the lower right corner.

As shown in Figure 2a, there is a slit in the comb structure. Therefore, if disturbances occur, such as a variance in temperature or unevenness of the substrate, the excess liquid which passes into the main channel from the reservoir can flow through the slit of the comb structure to the outside branches, and thus liquid leaking can be prevented at the tip of the print head. 

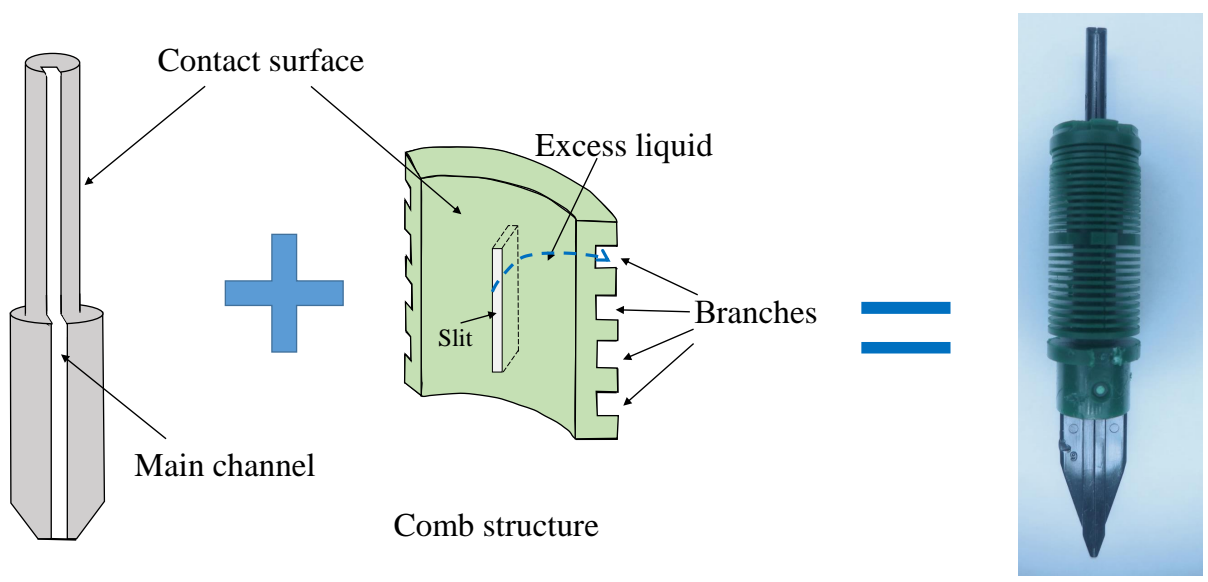

(a)

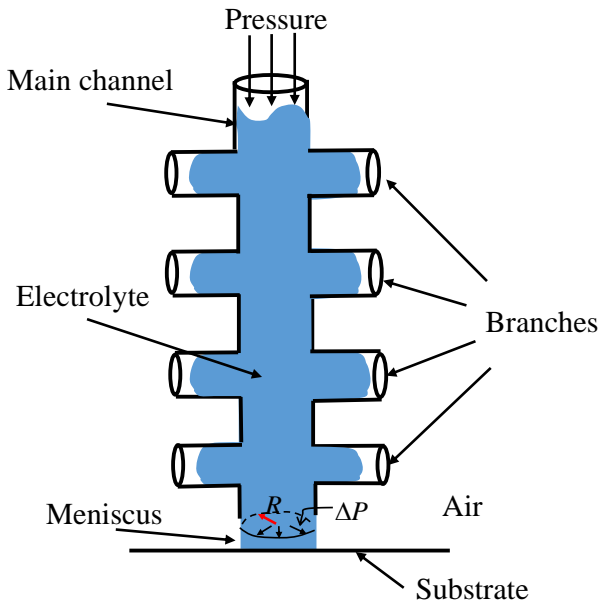

(b)

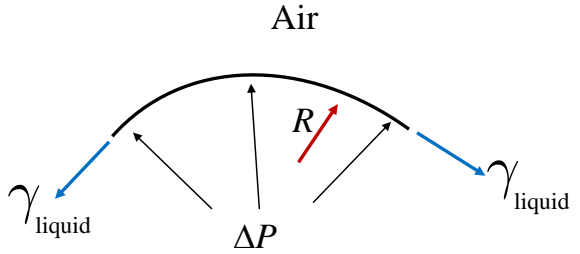

Liquid

(c)

Figure 2. (a) The detailed illustration of the main channel and the comb structure. Excess fluid can flow through the slit of the comb structure and to the outside branches. (b) Schematic illustration that how the branches can help to main a stable meniscus. (c) Force diagram of the meniscus at the interface between liquid and air.

This mechanism is further explained in Figure $2 b, c$. The branches shown in Figure $2 b$ act as the comb structure to store the excess liquid. If the meniscus shown in Figure $2 b$ is simplified to have a shape of cylinder with the radius of $R$, which is equal to the radius of the main channel, the key to maintaining a stable meniscus is the pressure difference between the inside and outside of the meniscus, which can be balanced by the surface tension $\gamma_{\text {liquid }}$ of the liquid, as illustrated in Figure $2 c$. The force equilibrium is expressed as

$$
\Delta P=\frac{\gamma_{\text {liquid }}}{R}
$$

If disturbances occur which causes a sudden increase of $\Delta P$, and the surface tension $\gamma_{\text {liquid }}$ is insufficient to balance this increase, liquid leaking would occur at the tip of the print head. On the other hand, if the radius $R$ is increased to enlarge the deposition area, which thus improves the deposition rate, the pressure provided by the surface tension $\gamma_{\text {liquid }}$ would decrease, and hence the stability of the meniscus is impaired. The branches shown in Figure $2 b$ not only store the excess liquid but also prevent the abrupt increase of the pressure difference $\Delta P$ at the tip of the print head. The effect of adding the branches was further analyzed by a simulation run in the commercial software Fluent.

As shown in Figure 3a,b, two 2D simulations were performed. There are no branches for the simulation in Figure 3a, and for the simulation in Figure 3b, eight branches are located symmetrically 
with respect to the main channel. The width of the branch channel is $20 \mu \mathrm{m}$ and the distance between adjacent branch channels is $30 \mu \mathrm{m}$. A constant pressure of $\Delta P=2000 \mathrm{~Pa}$ was applied at the inlet for both simulations shown in Figure 3a,b. The volume of fluid (VOF) approach in Fluent was used to track the interface of the liquid and air. Water was taken as the liquid in the simulations and the material parameters employed in the simulations are tabulated in Table 1 . For the phase field $\phi_{v}$ shown in Figure $3 \mathrm{a}, \mathrm{b}$, air is represented by $\phi_{v}=0$ while water is represented by $\phi_{v}=1$. Rectangular elements with the mesh size of $0.004 \mathrm{~mm} \times 0.004 \mathrm{~mm}$ are used and the time increment is $10^{-5} \mathrm{~s}$.

Figure $3 \mathrm{a}, \mathrm{b}$ shows the phase filed at a certain time for the case without and with eight branches, respectively. $\phi_{v}=0.8$ is chosen as the interface of water and air. It can be observed that the meniscus for the case without branches tends to flow outward while the meniscus for the case with eight branches is maintained in reasonable shape. The pressure from Point A to Point B (see Figure 3a) for the two cases is compared in Figure 3c. It can be clearly observed that the pressure for the case with eight branches is lower than that without branches, which implies the stability of the meniscus can be improved by adding the branches. It should be noted that the simulation with eight branches shown in Figure $3 \mathrm{~b}$ is employed to schematically illustrate that the branched structure is capable of maintaining a more stable meniscus. There are 24 branches for the real comb structure shown in Figure 2a. Therefore, the effect of maintaining a stable meniscus by the real comb structure is expected to be much better than that of the simulated structure with eight branches. As detailed in the following sections, several experiments were conducted to demonstrate the processing ability of the proposed design.

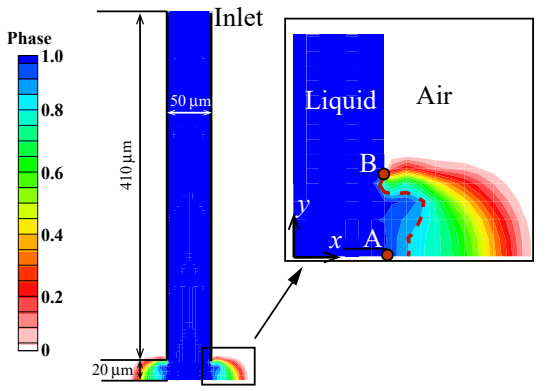

(a)

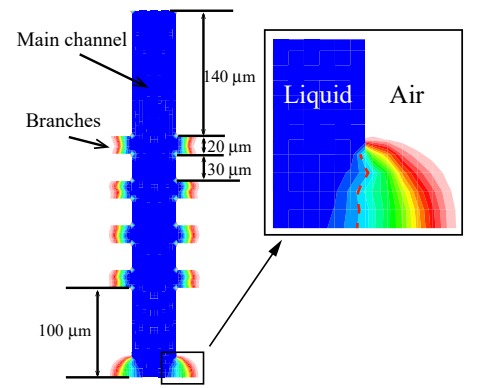

(b)

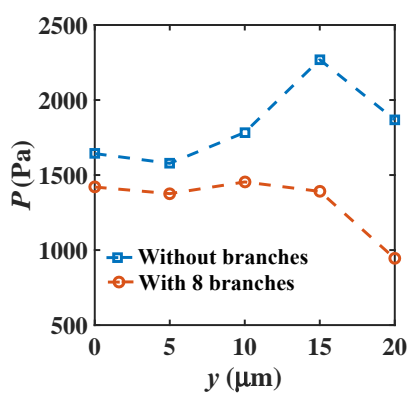

(c)

Figure 3. Simulations on the fluid flow in the main channel: (a) without branches; and (b) with eight branches. (c) The pressure along Point A to Point B shown in (a).

Table 1. Physical conditions of water and air [26].

\begin{tabular}{cccc}
\hline & Density $\left(\mathrm{kg} / \mathrm{m}^{3}\right)$ & Viscosity $($ Pa $\cdot \mathbf{s})$ & Surface Tension $(\mathrm{N} / \mathrm{m})$ \\
\hline Water & 988.2 & 0.001 & 0.072 \\
Air & 1.225 & $1.8 \times 10^{-5}$ & - \\
\hline
\end{tabular}

\section{Experiments}

\subsection{Materials}

The experiments were mainly conducted for the deposition of copper and the electrolytes were a mixture of $1 \mathrm{M} \mathrm{CuSO}_{4}$ and $0.005 \mathrm{M} \mathrm{H}_{2} \mathrm{SO}_{4}$ in deionized water with ratio 1:1 by volume. A nickel square was also printed to demonstrate the ability to print various materials. The electrolytes for nickel deposition were $113 \mathrm{gL}^{-1} \mathrm{NiSO}_{4} \cdot 6 \mathrm{H}_{2} \mathrm{O}, 30 \mathrm{gL}^{-1} \mathrm{NiCl}_{2} \cdot 6 \mathrm{H}_{2} \mathrm{O}$ and $23 \mathrm{gL}^{-1} \mathrm{H}_{3} \mathrm{BO}_{3}$ [21]. 


\subsection{Apparatus}

Three two-phase stepper motor stages (Zolix, Beijing) were employed to serve as the $x-, y-$, and $z$-stages. The stepper motor stages have the screw pith of $L=4 \mathrm{~mm}$ and step angle of $\alpha=1.8^{\circ}$ with $n$ $=20$ subdivisions, resulting in the positioning resolution of $L \alpha / 360 n=1 \mu \mathrm{m}$ in Cartesian coordinates.

Three materials, namely the polished brass plate which was composed of $67 \% \mathrm{Cu}$ and $33 \% \mathrm{Zn}$ with the surface roughness $\mathrm{Ra}=0.017 \mu \mathrm{m}$, the unpolished copper plate with $\mathrm{Ra}=0.19 \mu \mathrm{m}$, and the polyethylene terephthalate (PET), were tested to serve as the substrate. The surface roughness Ra, which was evaluated by the arithmetic average of the absolute values of the profile height deviations from the mean line within the evaluation length, was measured using a Mitutoyo SJ-210 surface roughness tester with an evaluation length of $1 \mathrm{~mm}$.

As shown in Figure 1a, a syringe was employed as the reservoir and a copper or nickel wire was inserted into the electrolyte to serve as the anode, while the substrate served as the cathode. The electrical potential was applied between the anode and cathode by a signal generator (Tektronix AFG3011C). The syringe with the print head was held onto the $z$-stage and the print head was lowered slowly until the tip touched the substrate where a current signal was recorded by a Keithley digital multimeter DMM7510. Then, the print head retracted $30 \mu \mathrm{m}$ to form a stable meniscus.

A two-electrode system without the reference electrode was used in the present study. Since the active area of the anode (counter electrode) was far larger than that of the cathode (working electrode), the polarization of the counter electrode was expected to be small [27]. The deposition voltage was tuned to make the corresponding current $\sim 0.3 \mathrm{~mA}$, which was reported as the optimal deposition current for copper in [22]. After a parametric study, the potential of $25 \mathrm{~V}$ between the anode and cathode was selected (the corresponding current recorded was $\sim 0.3 \mathrm{~mA}$ ). Both the constant $(25 \mathrm{~V})$ and pulsed potential $(25 \mathrm{~V}, 1 \mathrm{kHz}$, duty cycle $=0.3)$ were tested for copper deposition. For simplicity, a content potential of $25 \mathrm{~V}$ was also employed to print the nickel. It should be noted that the potential value selected was relatively larger than that reported in [25], in which a three-electrode system where a copper bar was used as the reference electrode was employed and a maximum potential of $6 \mathrm{~V}$ was applied between the reference electrode and the cathode. In the proposed design, the electrolyte needs to pass through the long and narrow main channel to the tip of the print head. As a result, the transport of the metal ions from the reservoir to the tip of the print head is limited due to the high width to height ratio of the main channel [28]. Therefore, the concentration of the metal ions through the long main channel is expected to be lower than that in the reservoir, which would increase the resistance of the whole circuit.

A commercially obtained fountain pen, as shown in Figure 4, was employed as the print head. The tip of the main channel was machined by a laser engraving machine (Branded Hans Laser) to control the width in the range of 50-300 $\mu \mathrm{m}$. The width of the slit in the comb structure was $\sim 400 \mu \mathrm{m}$. The material used for the main channel and the comb structure was acrylonitrile butadiene styrene plastic (ABS).

Scanning electron microscopy (SEM) images and energy-dispersive X-ray spectroscopy (EDX) element mapping were obtained at high vacuum with operating voltage of $20 \mathrm{kV}$. Elastic modulus of the printed structure was measured by the nanoindentation test on an Agilent Nano Indenter G200, and microhardness tests were performed on a Fischerscope HM200. 


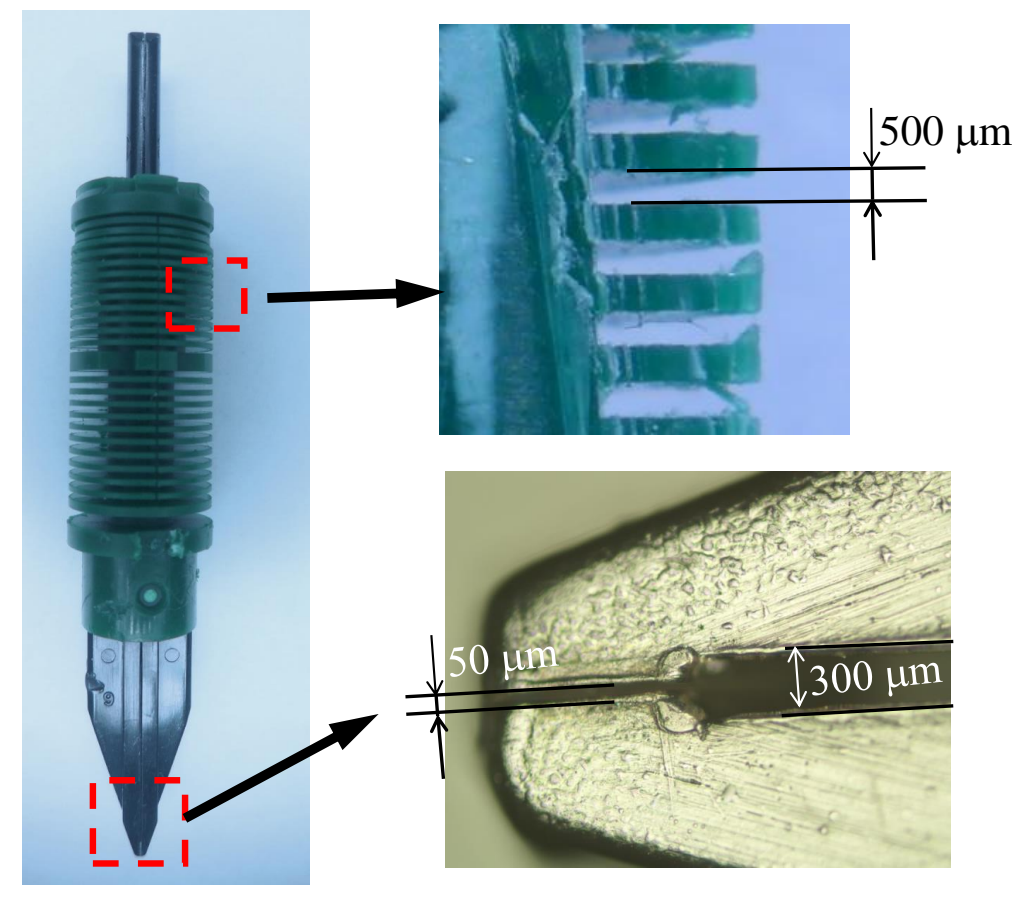

Figure 4. The dimensions of the print head.

\section{Results and Discussions}

\subsection{Printing Speed}

To determine the maximum appropriate printing speed for the proposed ECAM system that can be achieved with a relatively high resolution and uniform morphology, lines printed at various printing speeds on the brass substrate were investigated. For simplification and convenience regarding the influence of frequency and duty cycle of the pulsed potential, a constant potential was simply applied. An optical microscope was employed to measure the printed line width. Figure 5a shows the printed copper line width as a function of printing speed. It can be observed that the printed line width decreases in an approximately linear fashion as speed increases, which indicates that a higher speed leads to a higher resolution of the printed line. However, a printing speed that is too high results in a non-uniform printed line width along the printing direction and serrated boundaries. For instance, as shown in Figure 5a, the line printed at $500 \mu \mathrm{m} / \mathrm{s}$ is much more uniform than that printed at $1000 \mu \mathrm{m} / \mathrm{s}$. Further increasing the printing speed resulted in non-continuous printed lines, as shown in Figure $5 \mathrm{~b}$ for the speed of $3000 \mu \mathrm{m} / \mathrm{s}$. On the other hand, a printing speed that is too low not only impairs the deposition rate but also allows sufficient time for solvent (water) evaporation. As a result, the solute material (for instance, $\mathrm{CuSO}_{4}$ for copper deposition) readily precipitates on the substrate, such as for the line printed at $100 \mu \mathrm{m} / \mathrm{s}$. Consequently, in the present study, $500 \mu \mathrm{m} / \mathrm{s}$ was chosen as the optimal speed.

For the speed of $500 \mu \mathrm{m} / \mathrm{s}$, taking the diameter of the meniscus having the same dimension, i.e., $500 \mu \mathrm{m}$, and the current to be $\sim 0.3 \mathrm{~mA}$, then the corresponding current density was $0.15 \mathrm{~A} / \mathrm{cm}^{2}$, which was comparable to the values reported in $[3,21,29]\left(0.02-0.64 \mathrm{~A} / \mathrm{cm}^{2}\right)$. 


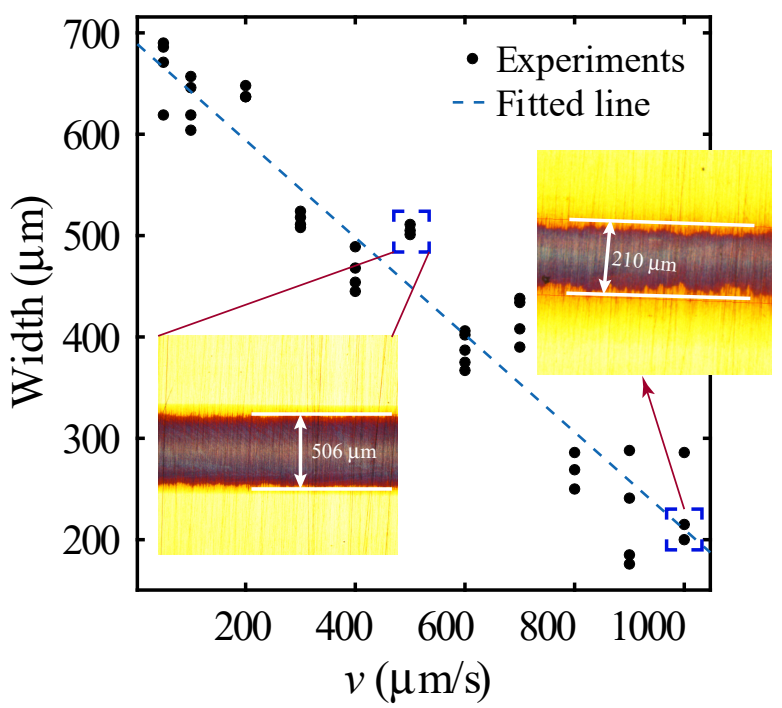

(a)

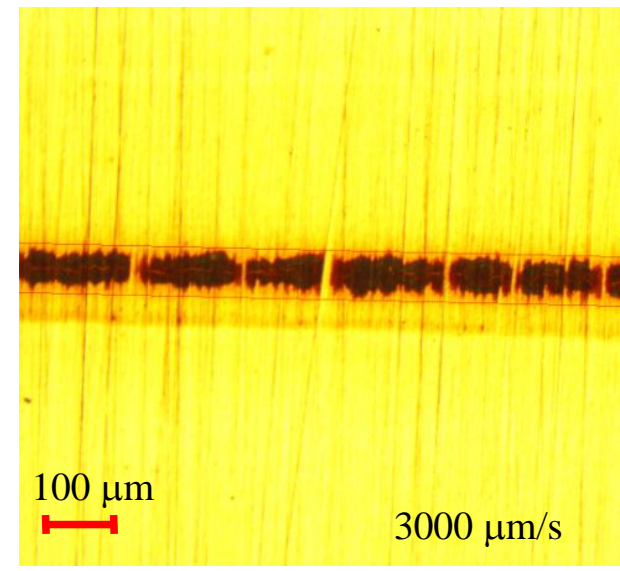

(b)

Figure 5. (a) Printed line width as a function of printing speed. (b) An optical microscope image of the printed line with the speed of $3000 \mu \mathrm{m} / \mathrm{s}$.

\subsection{Printing a Copper Square}

A copper square with the length of $2 \mathrm{~mm}$ was printed on the brass substrate. Two printing strategies, as shown in Figure $6 a, b$, with the constant and pulsed potential were investigated. The distance between two adjacent printing lines was $100 \mu \mathrm{m}$. For Printing Strategy 1 (see Figure 6a), the print head moves along the $x$-axis for all the layers; $\mathrm{Ra}_{x}$ in Figure $6 \mathrm{a}$ is the surface roughness measured along the $x$-direction, which is parallel to the printing direction, while $\mathrm{Ra}_{y}$ is the surface roughness measured along the $y$-direction, which is perpendicular to the printing direction. For Printing Strategy 2 (see Figure $6 \mathrm{~b}$ ), the printing direction rotates $90^{\circ}$ after printing each layer, and the surface roughness $\mathrm{Ra}$ is measured for both the $x$ - and $y$-directions. The Ra values shown in Figure $6 \mathrm{c}$ were averaged by measuring three samples, and, for each sample, at least five different locations were analyzed.

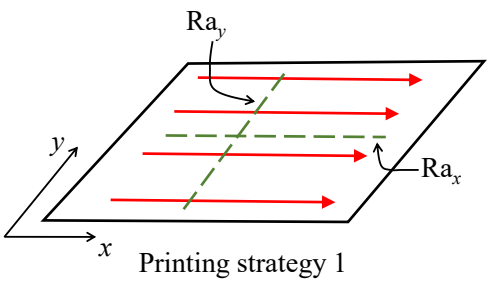

(a)

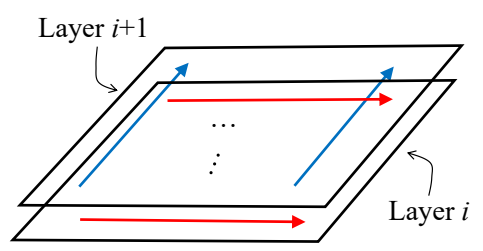

Printing strategy 2

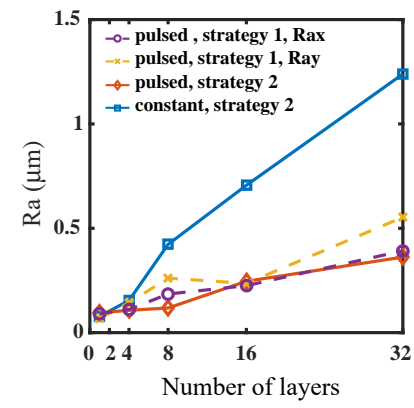

(c)

Figure 6. The average surface roughness, $\mathrm{Ra}$, was investigated for two printing strategies. (a) Printing Strategy 1 , where the print head moves along the $x$-axis for all layers. (b) Printing Strategy 2, where the printing direction rotates $90^{\circ}$ after printing each layer. (c) Ra as a function of the number of deposition layers and printing strategies under pulsed and constant potential.

Figure $6 \mathrm{c}$ shows that Ra increases with the increasing number of deposition layers. For Printing Strategy $1, \mathrm{Ra}_{x}$ is slightly smaller than $\mathrm{Ra}_{y}$, which suggests that the surface is smoother along the direction parallel to the printing direction than that along the perpendicular direction. In contrast, the square obtained by Printing Strategy 2 with a pulsed potential has the smallest Ra, which indicates that the surface roughness can be improved by applying the pulsed potential with varying the printing directions during the process. The advantage of Printing Strategy 2 compared to Printing Strategy 1 is 
that the upper-layer material can be filled into the gap and hole of the lower layer during the printing process, therefore some uneven areas can be repaired and the surface flatness is improved. The surface roughness shown in Figure 6c was measured using a Mitutoyo SJ-210 surface roughness tester with an evaluation length of $1 \mathrm{~mm}$.

Figure 7a shows the printed square by depositing two layers on the brass substrate with Printing Strategy 2 and the pulsed potential. The yellow part at the boundary is believed that some electrolytes were accumulated at the boundary during printing and the zinc element in the brass substrate was dissolved in the electrolytes and then deposited on the substrate. This was confirmed by using an unpolished copper substrate and, repeating the same process for printing the square, the yellow part was no longer observed, as shown in Figure $7 \mathrm{~b}$. The proposed ECAM system also worked well on both the polished and unpolished substrates, and the stability of the meniscus was not affected. Further measurements showed that the square printed on the polished substrate resulted in a smoother printed surface $(\mathrm{Ra}=0.036 \mu \mathrm{m})$ than that on the unpolished substrate $(\mathrm{Ra}=0.233 \mu \mathrm{m})$. This is mainly because the actual surface area of the rough surface is larger than that of the smooth surface, therefore the current density on the rough surface is relatively small. If the local current density were not sufficient for the deposition of metal ions, there would be no metals deposited on that region. Hence, due to the worse uniformity of current density on the rough surface, the corresponding roughness of the printed structures is higher.

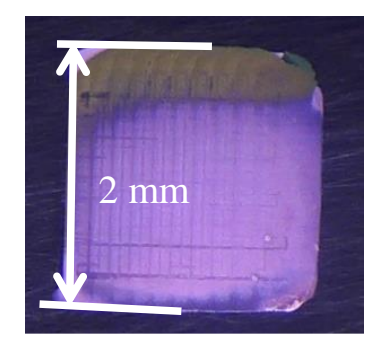

(a)

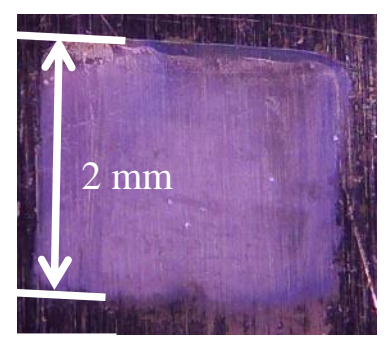

(b)

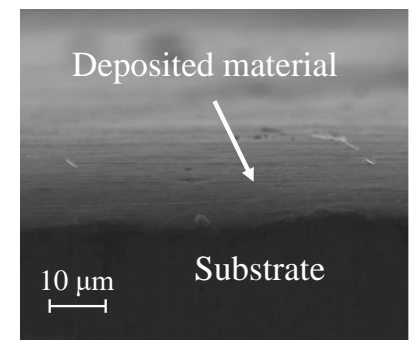

(c)

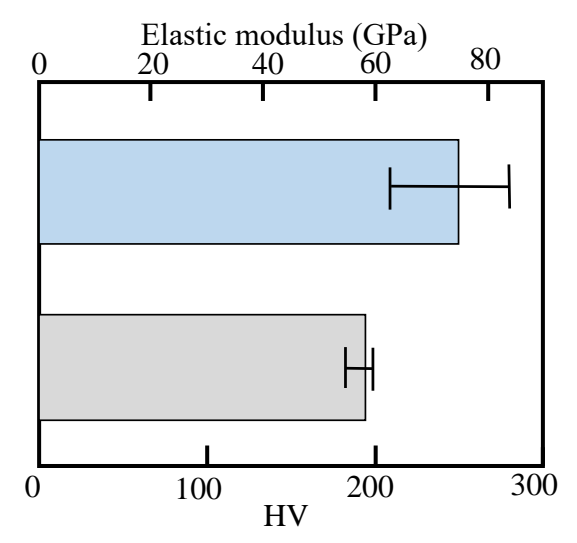

(d)

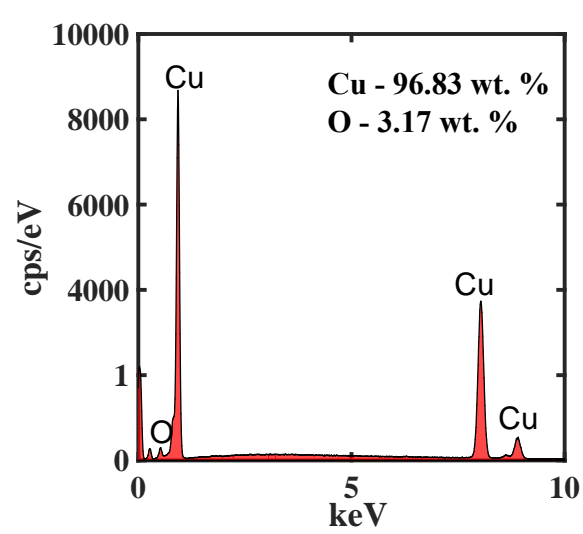

(e)

Figure 7. (a) A copper square printed on the polished brass substrate for two layers. (b) A copper square printed on the unpolished copper substrate for two layers. (c) SEM image of the side view of a copper square printed on the brass substrate for 32 layers. (d) Elastic modulus and Vickers hardness for the printed copper square. (e) EDX of the printed copper square.

The side view for the square printed on the brass substrate for 32 layers is shown in Figure 7c, illustrating the dense morphology and height of $\sim 12 \mu \mathrm{m}$. As the printing speed was $500 \mu \mathrm{m} / \mathrm{s}$ with 22 passes (each pass was $2 \mathrm{~mm}$ ) for printing each layer, the total time for printing every layer was $88 \mathrm{~s}$. Hence, Figure $7 \mathrm{c}$ suggests the thickness for each pass is $\sim 375 \mathrm{~nm}$. This implies that the printing process has an exceptionally high $z$-height resolution, making it potentially useful for fabricating functional electronics such as thin-film sensors. 
The elastic modulus $E$ and Vickers hardness HV of the printed copper square on the polished brass substrate were measured by the nanoindentation and microhardness tests, respectively. Six points were tested and the results are shown in Figure $7 \mathrm{~d}$. It can be observed that the average elastic modulus of the printed copper is $\sim 75 \mathrm{GPa}$, while the average Vickers hardness is $\sim 200 \mathrm{HV}$. As a reference, Chen et al. [25] reported that the hardness of their printed copper structures ranged from 184 to $228 \mathrm{HV}$ and the hardness of copper used in circuit bonding wires can range from 50 to $176 \mathrm{HV}$ [30]. The processes for obtaining the elastic modulus and Vickers hardness are explained in Appendix A. The formation of pure elemental copper for the printed square on the polished brass substrate was confirmed by EDX, as shown in Figure 7e. The deposited copper of the test point can be as high as $96.83 \mathrm{wt} \%$ with $3.17 \mathrm{wt} \%$ of oxygen. Further SEM study showed the grain size of the printed square was as small as $\sim 1 \mu \mathrm{m}$.

\subsection{Printing Various Copper Structures}

Three letters, "S", " $Y$ ", and " $U$ ", were separately printed from corresponding computer-aided design (CAD) models with pulsed potential and the image of a combination of the printed letters featuring the pattern "SYSU" is shown in Figure 8a. As the letters were printed separately, to make a uniform background, the substrate is removed and only the letters are shown in Figure 8a. A typical SEM image of the letter " $U$ " is shown in Figure $8 b$, illustrating the dense copper morphology without noticeable dendrites. Figure $8 \mathrm{c}$ is a close-up of the white boxed area in Figure $8 \mathrm{~b}$ and clearly shows that the printed letter has a smooth surface. Multiple passes were applied for printing each letter and the printing time was $\sim 45 \mathrm{~min}$. SEM images of the printed letter " $Y$ " (Figure $7 \mathrm{~d}$ ) indicates that the printed copper structures are polycrystalline with the grain size as small as $\sim 1 \mu \mathrm{m}$. The letter " $Y$ " shown in the boxed area in Figure $7 \mathrm{~d}$ is an unedited image without removing the substrate.
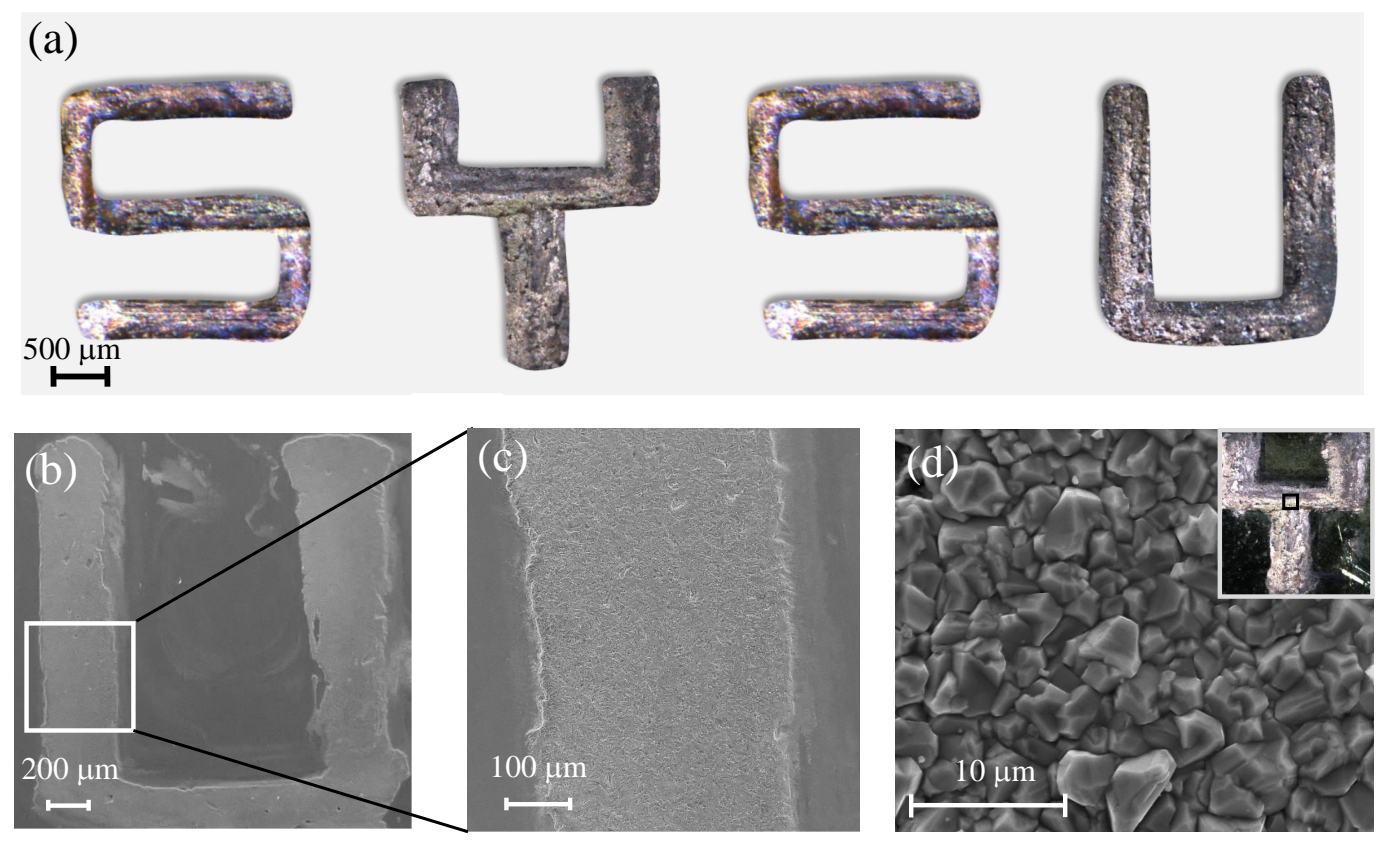

Figure 8. (a) Images of printed structures featuring the letters "SYSU." Only the letters without the substrate are shown to make an uniform background. (b) A typical SEM image of the letter " $U$ "; the zoomed-in view of the white boxed area is shown in (c). (d) SEM image of the white boxed area in letter " $\mathrm{Y}$ ".

The letter " $U$ " printed under pulsed and constant potential are also compared in Figure 9a,b. The SEM images show that the pulsed potential results in denser morphologies with sharp boundaries (see Figure 9a), whereas the structures printed under the constant potential have a porous morphology (see 
Figure $7 \mathrm{~b}$ ). This is mainly because the pulsed potential can provide a higher current density, which favors the initiation of grain nuclei and leads to finer-grained deposition [31]. Again, The two letters "U" shown in the boxed area of Figure 9a,b are unedited images without removing the substrate.
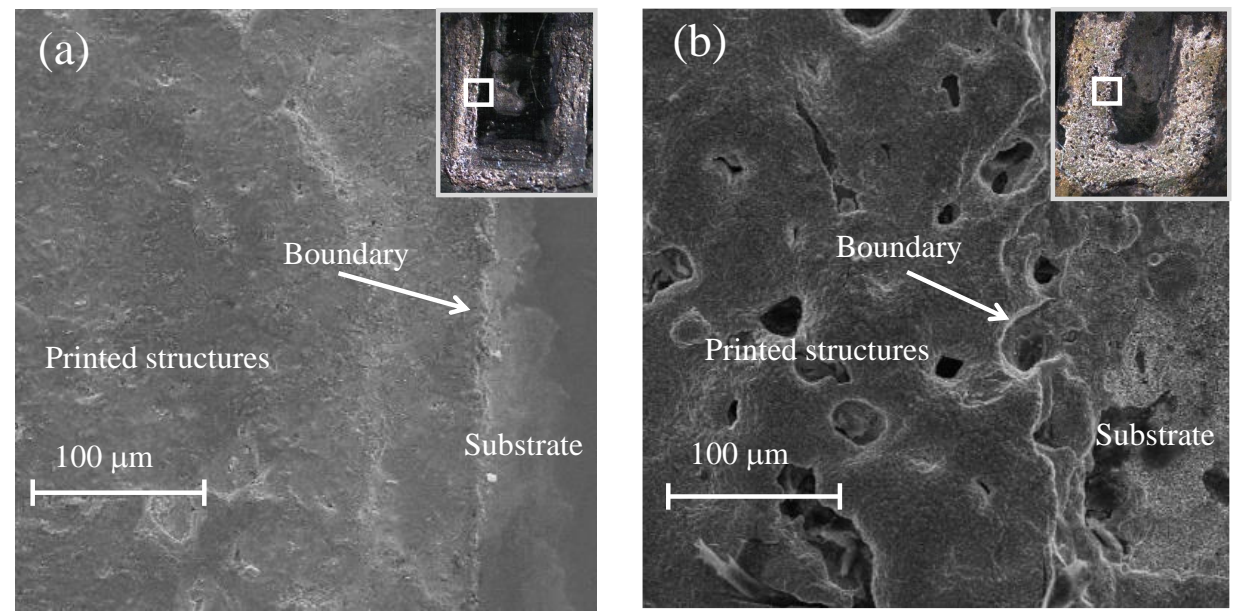

Figure 9. SEM images of the letter "U" printed under (a) pulsed and (b) constant potential.

A few attempts were also made to print free standing copper pillars, which are shown in Figure 10. Figure 10a,b shows the printed copper pillars with slopes of $\tan \alpha=1$ and 2 , respectively, where $\alpha$ is the angle with respect to the $x$-axis. Figure 10c is a printed vertical pillar followed by a pillar with the slope $\tan \alpha=3$, and Figure $10 \mathrm{~d}$ is a mixed structure with the overhanging pillars. The red arrows indicate the movement paths of the print head, and the numbers in Figure 10d represent the printing path sequence. The printed pillars with slopes $\tan \alpha$ in Figure $10 \mathrm{a}-\mathrm{c}$ were achieved by alternately moving the print head a distance of $d=3 \mu \mathrm{m}$ along the $x$-axis and $d \cdot \tan \alpha$ along the $z$-axis. The movement paths in Figure 10d were also realized by controlling the movement distances of the $x$ - and $z$-stages via a computer program. The speed of the print head was controlled to a low value $(3 \mu \mathrm{m} / \mathrm{s})$ for both the $x$ - and $z$-stages. The diameter of the printed pillars in Figure 10 is $\sim 50 \mu \mathrm{m}$, and dendritic morphologies can be observed in all structures. As explained in [25], when printing structures such as those in Figure 8, movement of the print head within the $x-y$ plane results in mechanical removal of the dendrites. However, for the structures shown in Figure 10, without the aid of mechanical removal, dendrites are easily formed due to preferential deposition at the center of the meniscus, which could be improved by applying an appropriate type of potential [4,25]. Nevertheless, Figure 10 demonstrates the ability of the proposed ECAM system to print structures with small overhanging angles.
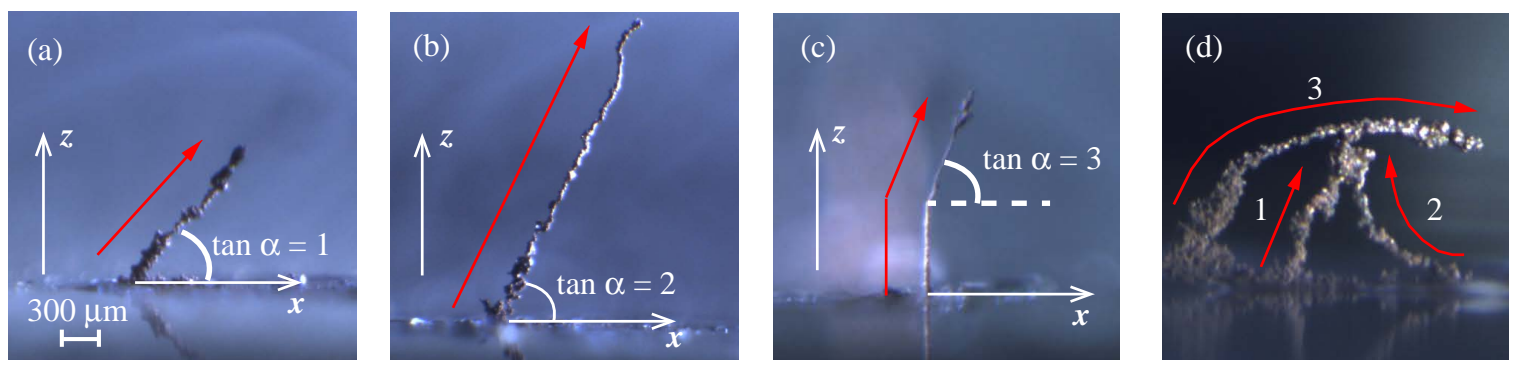

Figure 10. Images of 3D-printed Cu structures with different shapes. Red arrows indicate the movement paths of the print head. (a) A tilted pillar with slope $\tan \alpha=1$, where $\alpha$ is the angle with respect to the $x$-axis. (b) A tilted pillar with slope $\tan \alpha=2$. (c) A vertical pillar followed by a tilted pillar with slope $\tan \alpha=3$. (d) Mixed structure with overhanging features. The numbers indicate the sequence of the printing path. 


\subsection{Printing a Copper Circuit on the PET Substrate}

A circuit printed on the PET substrate was attempted to present a potential application with the proposed ECAM system. As the PET substrate was non-conductive, a thin copper film was first deposited on the PET substrate as the seed layer through the vacuum vapor plating technology. The thickness of the seed layer was $150 \mathrm{~nm}$. This thickness ensured good chemical and mechanical properties of the deposited copper [32] and good adhesion between the substrate and the seed layer [24]. The proposed printing process was then performed on the seed layer to fabricate the designed patterns. Finally, a chemical etching process was employed to remove the seed layer and clean the substrate. The etching solution was composed of $0.4 \mathrm{M}$ hydrochloric acid and $0.07 \mathrm{M} \mathrm{FeCl}_{3}$ and the etching process lasted $10 \mathrm{~s}$. Before depositing the seed layer, the substrate was cleaned by rinsing with ethanol and ultrapure water and subsequent 10 min plasma. As shown in Figure 11, a "pulse signal" pattern was printed on the PET substrate. Multiple passes were performed for printing the "pulse signal" pattern and the printing time was $\sim 1 \mathrm{~h}$. This example is shown as a proof-of-concept. Further investigations are needed to improve the quality of the printed circuit by optimizing the processing parameters and testing the corresponding electrical and mechanical properties.

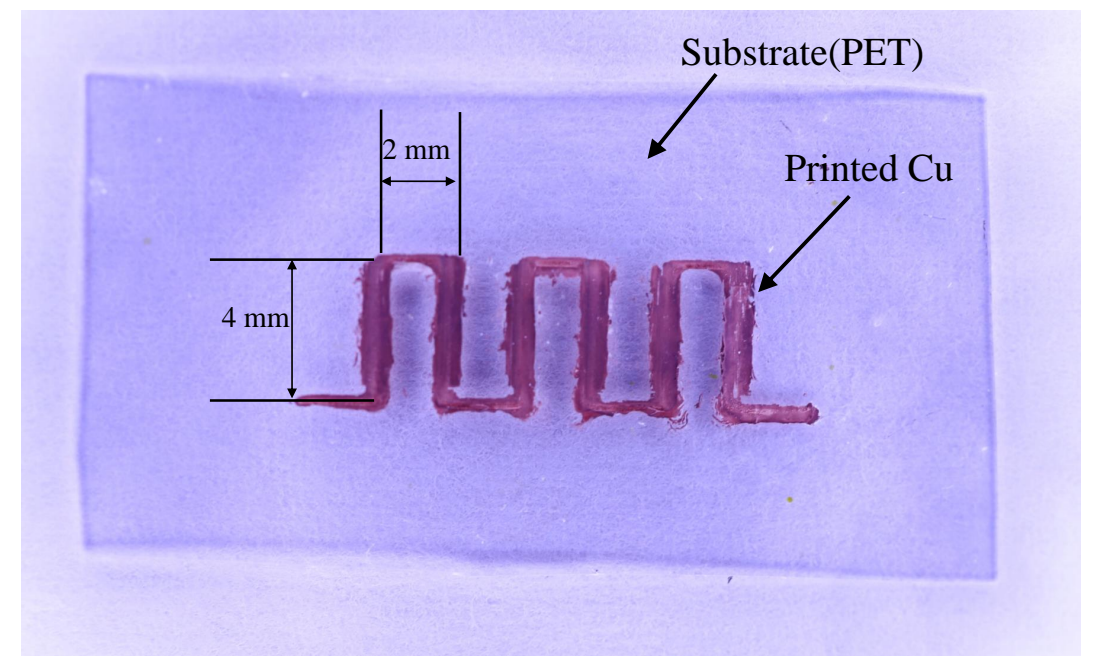

Figure 11. A "pulse signal" pattern printed on the PET substrate.

\subsection{Printing a Nickel Square}

A nickel square was finally printed with the same configurations as printing the copper. The square was printed using Printing Strategy 2 (see Figure 6b) for 16 layers. The SEM image of the printed nickel square is shown in Figure 12a. The formation of the nickel was further confirmed by testing a small region of the printed square using the EDX element mapping, as shown in Figure 12b. The printed nickel was $86.47 \mathrm{wt} \%$. The elements of copper and zinc tabulated in Figure $12 \mathrm{~b}$ were believed to come from the brass substrate. The surface roughness Ra of the printed nickel square was $0.141 \mu \mathrm{m}$. Figure 12 demonstrates various materials can be printed by the proposed ECAM system. Future studies may incorporate the electrolytes for printing the nickel and copper and control the depositing sequence of different elements by manipulating the applied potential. 


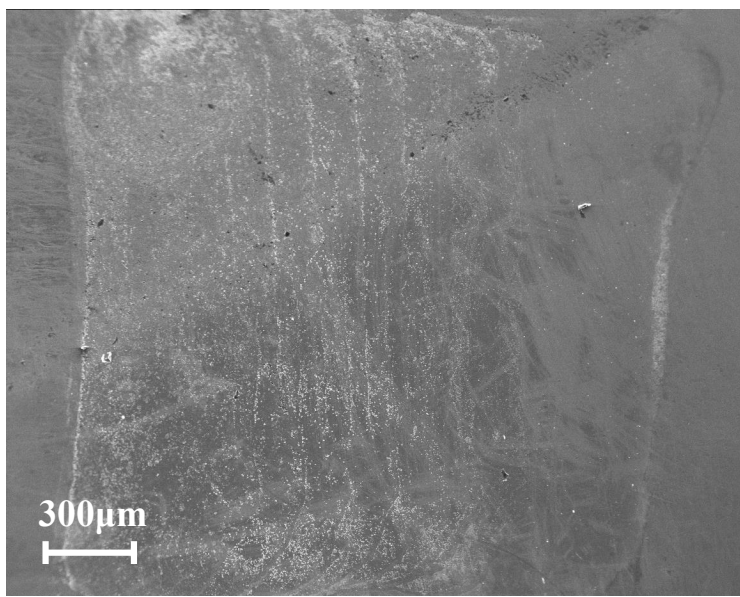

(a)

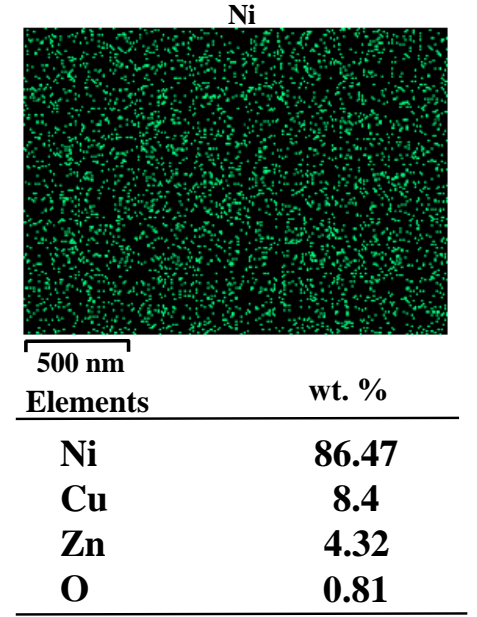

(b)

Figure 12. (a) The printed nickel square. (b) EDX element mapping for a small region of the printed nickel square and the corresponding wt. $\%$ of the detected elements.

\section{Conclusions}

This work presents the novel design of a low-cost ECAM system for printing mesoscale metal parts. With the proposed microfluidic system, i.e., the fountain pen feed system, which is composed of a semi-open main channel and comb structure, a stable mesoscale meniscus can be obtained during the printing process without liquid leaking or meniscus breaking. Two materials, copper and nickel, were attempted to print. It is believed that some other metals that can be electrodeposited from aqueous electrolytes, such as Ag and Ti, can be printed through the same ECAM system. The investigation of the surface roughness of the printed structures shows that the surface smoothness can be controlled by manipulating the applied potential and printing strategy. Various structures were also printed and an example of a copper circuit printed on the non-conductive PET substrate was achieved to provide a possible application using the proposed ECAM system. The presented work demonstrates the possibility to control the meniscus and electrolyte flow by designing an appropriate microfluidic system and provides a foundation for future work on designing more sophisticated microfluidic systems to achieve higher printing precisions and satisfactory material properties for the fabrication of functional electronics.

Author Contributions: Conceptualization, Y.Y.; validation, P.L., Y.G., Y.W., and J.C.; formal analysis, P.L. and Y.G.; investigation, P.L. and Y.G.; writing-original draft preparation, P.L. and Y.Y.; writing-review and editing, P.L., Y.G., Y.W., J.C., and Y.Y.; and supervision, Y.Y. All authors have read and agreed to the published version of the manuscript.

Funding: This research received no external fund.

Conflicts of Interest: The authors declare no conflict of interest.

\section{Appendix A.}

\section{Appendix A.1. Nanoindentation Test}

A load of $0.78 \mathrm{mN}$ was applied on the sample for the nanoindentation test with a Berkovich indenter and a typical loading and unloading curve is shown in Figure A1. The unloading curve was fitted by

$$
P_{u}=a\left(h-h_{f}\right)^{m}
$$


where $a, h$ and $h_{f}$ are the fitting parameters, which were fitted using MATLAB in the present study. Then, the contact stiffness was established as

$$
S=\left.\frac{d P_{u}}{d h}\right|_{h}=h_{\max }=m a\left(h_{\max }-h_{f}\right)^{m-1},
$$

where $h_{\max }$ is the maximum indentation depth.

The elastic modulus and hardness were calculated by the Oliver-Pharr method and were expressed as

$$
\frac{1}{E_{r}}=\frac{1-v^{2}}{E}+\frac{1-v_{i}^{2}}{E_{i}}
$$

where $E_{i}$ and $v_{i}$ are the elastic modulus (1140 GPa) and Poisson's ratio (0.07), respectively, of the indenter, and $E$ and $v$ are the elastic modulus and Poisson's ratio, respectively, of the test material. The modulus $E_{r}$ was expressed as

$$
E_{r}=\frac{\sqrt{\pi}}{2} \cdot \frac{S}{\sqrt{A}}
$$

where $S$ is the contact stiffness and $A$ is the projected contact area. The projected contact area A was calculated by

$$
A=C_{1} h_{c}^{2}+C_{2} h_{c}+C_{3} h_{c}^{1 / 2},
$$

where $C_{1}=23.97, C_{2}=391.7$, and $C_{3}=2018.2$, which were calibrated by the test machine. The contact depth $h_{c}$ was estimated by

$$
h_{c}=h_{\max }-\varepsilon \frac{P_{u}}{S},
$$

where $\varepsilon$ is a constant, which is related to the geometry of the indenter. For a Berkovich indenter, $\varepsilon=0.75$.

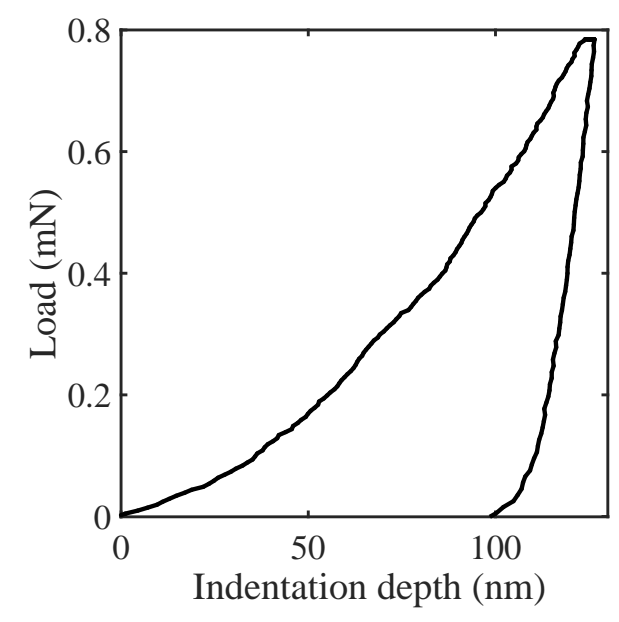

Figure A1. The loading and unloading curve from the nanoindentation test.

For all the material points tested, the ratio of final indentation depth $h_{f}$ to the maximum indentation depth $h_{\max }\left(h_{f} / h_{\max }\right)$ was $\sim 0.83$, which indicated that slight pile-up may have occurred under nanoindentation. As a result, the measured elastic modulus and hardness may be slightly overestimated. As reported by Moharrami and Bull [33], for the indentation depth obtained in the present study, the maximum overestimation for the elastic modulus was $8 \%$. 
Appendix A.2. Vickers Hardness Test

A maximum load of $F=1000 \mathrm{mN}$ was applied for the Vickers hardness test with the maximum indentation depth of $\sim 4.6 \mu \mathrm{m}$. The loading and unloading time were both $20 \mathrm{~s}$ and the dwell time was $10 \mathrm{~s}$. For the Vickers hardness test, a diamond indenter with the tip angle of $136^{\circ}$ was employed and the averaged diagonal length $\mathrm{d}(\mu \mathrm{m})$ of the contact area was measured to calculate the Vickers hardness HV by

$$
\mathrm{HV}=\frac{0.1891 F}{d^{2}} \times 10^{3}
$$

\section{References}

1. Yap, C.Y.; Chua, C.K.; Dong, Z.L.; Liu, Z.H.; Zhang, D.Q.; Loh, L.E.; Sing, S.L. Review of selective laser melting: Materials and applications. Appl. Phys. Rev. 2015, 2, 041101. [CrossRef]

2. Gong, X.; Anderson, T.; Chou, K. Review on powder-based electron beam additive manufacturing technology. In Proceedings of the ASME/ISCIE 2012 International Symposium on Flexible Automation, St. Louis, MO, USA, 18-20 June 2012; pp. 507-515.

3. Behroozfar, A.; Daryadel, S.; Morsali, S.R.; Moreno, S.; Baniasadi, M.; Bernal, R.A.; Minary-Jolandan, M. Microscale 3D printing of nanotwinned copper. Adv. Mater. 2018, 30, 1705107. [CrossRef] [PubMed]

4. Seol, S.K.; Kim, D.; Lee, S.; Kim, J.H.; Chang, W.S.; Kim, J.T. Electrodeposition-based 3D printing of metallic microarchitectures with controlled internal structures. Small 2015, 11, 3896-3902. [CrossRef] [PubMed]

5. Francois, M.; Sun, A.; King, W.; Henson, N.; Tourret, D.; Bronkhorst, C.; Carlson, N.; Newman, C.; Haut, T.; Bakosi, J.; et al. Modeling of additive manufacturing processes for metals: Challenges and opportunities. Curr. Opin. Solid State Mater. Sci. 2017, 21, 198-206. [CrossRef]

6. Yang, Y.; Knol, M.; van Keulen, F.; Ayas, C. A semi-analytical thermal modelling approach for selective laser melting. Addit. Manuf. 2018, 21, 284-297. [CrossRef]

7. Yang, Y.; van Keulen, F.; Ayas, C. A computationally efficient thermal model for selective laser melting. Addit. Manuf. 2020, 31, 100955. [CrossRef]

8. Sundaram, M.M.; Kamaraj, A.B.; Kumar, V.S. Mask-less electrochemical additive manufacturing: A feasibility study. J. Manuf. Sci. Eng. 2015, 137, 021006. [CrossRef]

9. Madden, J.D.; Hunter, I.W. Three-dimensional microfabrication by localized electrochemical deposition. J. Microelectromechanical Syst. 1996, 5, 24-32. [CrossRef]

10. Said, R. Localized electro-deposition (LED): The march toward process development. Nanotechnology 2004, 15, S649. [CrossRef]

11. Je, J.H.; Kim, J.M.; Jaworski, J. Progression in the fountain pen approach: From 2D writing to 3D free-form micro/nanofabrication. Small 2017, 13, 1600137. [CrossRef]

12. $\mathrm{Hu}, \mathrm{J} . ; \mathrm{Yu}, \mathrm{M} . \mathrm{F}$. Meniscus-confined three-dimensional electrodeposition for direct writing of wire bonds. Science 2010, 329, 313-316. [CrossRef] [PubMed]

13. Kim, K.H.; Moldovan, N.; Espinosa, H.D. A nanofountain probe with sub-100 nm molecular writing resolution. Small 2005, 1, 632-635. [CrossRef] [PubMed]

14. Kim, K.; Ke, C.; Moldovan, N.; Espinosa, H. Massively parallel multi-tip nanoscale writer with fluidic capabilities-fountain pen nanolithography (FPN). In Proceedings of the 4th International Symposium on MEMS and Nanotechnology, Charlotte, NC, USA, 2-4 June 2003; pp. 235-238.

15. Wu, B.; Ho, A.; Moldovan, N.; Espinosa, H.D. Direct deposition and assembly of gold colloidal particles using a nanofountain probe. Langmuir 2007, 23, 9120-9123. [CrossRef] [PubMed]

16. Loh, O.; Lam, R.; Chen, M.; Moldovan, N.; Huang, H.; Ho, D.; Espinosa, H.D. Nanofountain-probe-based high-resolution patterning and single-cell injection of functionalized nanodiamonds. Small 2009, 5, 1667-1674. [CrossRef]

17. Moldovan, N.; Kim, K.; Espinosa, H.D. A multi-ink linear array of nanofountain probes. J. Micromech. Microeng. 2006, 16, 1935. [CrossRef]

18. Hirt, L.; Grüter, R.R.; Berthelot, T.; Cornut, R.; Vörös, J.; Zambelli, T. Local surface modification via confined electrochemical deposition with FluidFM. Rsc Adv. 2015, 5, 84517-84522. [CrossRef] 
19. Falola, B.D.; Suni, I.I. Low temperature electrochemical deposition of highly active elements. Curr. Opin. Solid State Mater. Sci. 2015, 19, 77-84. [CrossRef]

20. Reiser, A.; Lindén, M.; Rohner, P.; Marchand, A.; Galinski, H.; Sologubenko, A.S.; Wheeler, J.M.; Zenobi, R.; Poulikakos, D.; Spolenak, R. Multi-metal electrohydrodynamic redox 3D printing at the submicron scale. Nat. Commun. 2019, 10, 1-8. [CrossRef]

21. Chen, X.; Liu, X.; Ouyang, M.; Chen, J.; Taiwo, O.; Xia, Y.; Childs, P.R.; Brandon, N.P.; Wu, B. Multi-metal 4D printing with a desktop electrochemical 3D printer. Sci. Rep. 2019, 9, 3973. [CrossRef]

22. Ambrosi, A.; Webster, R.D.; Pumera, M. Electrochemically driven multi-material 3D-printing. Appl. Mater. Today 2020, 18, 100530. [CrossRef]

23. Wang, P.; Roberts, R.; Ngan, A. Direct microfabrication of oxide patterns by local electrodeposition of precisely positioned electrolyte: The case of Cu2O. Sci. Rep. 2016, 6, 1-9. [CrossRef] [PubMed]

24. Kim, H.; Kim, J.G.; Park, J.W.; Chu, C.N. Selective copper metallization of nonconductive materials using jet-circulating electrodeposition. Precis. Eng. 2018, 51, 153-159. [CrossRef]

25. Chen, X.; Liu, X.; Childs, P.; Brandon, N.; Wu, B. A low cost desktop electrochemical metal 3D printer. Adv. Mater. Technol. 2017, 2, 1700148. [CrossRef]

26. Juggurnath, D.; Dauhoo, M.; Elahee, M.; Khoodaruth, A.; Osowade, A.; Olakoyejo, O.; Obayopo, S.; Adelaja, A. Simulations of air-water two-phase flow in an inclined pipe. In Proceedings of the 13th International Conference on Heat Transfer, Fluid Mechanics and Thermodynamics, Portoroz, Slovenia, 17-19 July 2017; pp. $77-84$.

27. Winquist, F.; Krantz-Rülcker, C.; Lundström, I. A miniaturized voltammetric electronic tongue. Anal. Lett. 2008, 41, 917-924. [CrossRef]

28. Hoshyargar, V.; Nezameddin Ashrafizadeh, S.; Sadeghi, A. Diffusioosmotic flow in rectangular microchannels. Electrophoresis 2016, 37, 809-817. [CrossRef]

29. Lu, L.; Chen, X.; Huang, X.; Lu, K. Revealing the maximum strength in nanotwinned copper. Science 2009, 323, 607-610. [CrossRef]

30. Yeung, J.; Keong, L.C. Hardness measurement of copper bonding wire. Procedia Eng. 2014, 75, 134-139. [CrossRef]

31. Chandrasekar, M.; Pushpavanam, M. Pulse and pulse reverse plating-Conceptual, advantages and applications. Electrochim. Acta 2008, 53, 3313-3322. [CrossRef]

32. Pan, Y.; Liu, Y.; Wang, T.; Lu, X. Effect of a Cu seed layer on electroplated Cu film. Microelectron. Eng. 2013, 105, 18-24. [CrossRef]

33. Moharrami, N.; Bull, S. A comparison of nanoindentation pile-up in bulk materials and thin films. Thin Solid Film. 2014, 572, 189-199. [CrossRef]

(c) 2020 by the authors. Licensee MDPI, Basel, Switzerland. This article is an open access article distributed under the terms and conditions of the Creative Commons Attribution (CC BY) license (http://creativecommons.org/licenses/by/4.0/). 